\title{
An analysis of Approaches for Desktop Virtualization and Challenges
}

\author{
Rupesh Kumar, Arun Kumar Yadav, H N Verma
}

Department of Computer Science and Engineering, ITM University, Gwalior, Madhya Pradesh, India

\begin{abstract}
Article Info

Volume 7, Issue 4

Page Number : 600-612

Publication Issue :

July-August-2021

\section{Article History}

Accepted : 12 Aug 2021

Published : 23 Aug 2021

In the Information Technology world, cloud computing technology offering unlimited amount of IT resources and services to end users over the internet on pay-per-use basis. End users are accessing the cloud services on their mobile or personal computers. Service providers are upgrading their services very frequently to enhance the services, and to use their upgraded services, end users are also required to update the specification of their devices. But it will be very costly for the end users to upgrade their devices for high specification to use the enhanced services. Desktop Virtualization is an extensive technology of cloud services. It is the new concept, in which users can access the virtual desktop of required specifications, software and operating system on their old devices anytime and anywhere. With the help of desktop virtualization, users will be benefited by avoiding the cost of frequent upgradation of mobile or personal computer system. Desktop virtualization technology is proving to be a boon for large and small organizations who have to upgrade their computer system with new technology, which is a very costly and challenging process. Desktop virtualization avoids upgrading the hardware of the client machine repeatedly. It allows us to access all applications and data at a low cost on our old machine. This paper presents the comparative analysis of various approaches for desktop virtualization and various challenges which required the solution. Analysis presented in paper has been done based on various performance parameters which will provide the end users low-cost cloud services and best performance on their mobile or personal computers.
\end{abstract}

Keywords : Desktop virtualization, Low-cost service, Security, Resource utilization, Power consumptions.

\section{INTRODUCTION}

One of the most essential technologies in the IT environment is cloud computing, and it is emerging vastly and no doubt it is the next generation technology where humans will be using anywhere and anytime. Cloud computing provides on demand IT services such as virtual storage for private data, software services, computing services are all easily delivered to the cloud client with the help of virtualization which the client can access all these services at any time and any place over the internet.

Copyright: @ the author(s), publisher and licensee Technoscience Academy. This is an open-access article distributed under the terms of the Creative Commons Attribution Non-Commercial License, which permits unrestricted non-commercial use, distribution, and reproduction in any medium, provided the original work is properly cited 


\section{A. Cloud Computing}

Cloud computing [1], [2], [3] is the best computing business model in the IT world based on the concept of virtualization [4] and shared infrastructure [5]. In this model, we install a large centralized data centre, in which all the data is stored. This data centre is also called a server. This server is connected to the client with the help of the internet and the client can get its data at any time and any place. There are many organizations that provide cloud services. This organization includes names like Google, Apple, Microsoft Azure, Amazon etc. which have made IT business easier by providing cloud services. Grid computing, utility computing and autonomic computing are the three factors of cloud computing and it is based on all these three factors.

\section{B. Types of Cloud Computing}

Public cloud, private cloud, community cloud and hybrid cloud are all types of cloud computing [6]. All these are mentioned in detail in the paragraph below1) Public Cloud: Public cloud provides some services such as virtual storage, applications, files etc. to the general public. Which works on the standard cloud framework. Organizations like Amazon, Google, and Microsoft etc. provide services such as Public Cloud to the general public. A public cloud is generally least expensive but less secure. Amazon Web Services (AWS) and Google App Engine (Gmail) are the best examples of public cloud.

2) Private Cloud: A Private cloud is a pay service that includes services such as storage, applications, and files. Private cloud provides more security to its clients, because of its implementation and control within the IT department and corporate firewalls. Organizations may build their own private cloud also called a data centre on top of Hyper-V, VMware, and virtual private network (VPN) technologies. Which we also call a data centre. For example, State Bank of India (SBI) has built a private cloud named Meghadoot that works on VMware technology to make its functionality faster, simpler, and to use all of its resources at a lower cost.

3) Community Cloud: A Community cloud is another form of private cloud in which many governments and private organizations share their similar requirements and concerns on the same platform to achieve their business objective. Which mainly includes concerns such as security, compliance and jurisdiction, joint project, application etc. Community cloud services are provided by most third parties at low cost with more security than public cloud to their clients.

4) Hybrid Cloud: Hybrid cloud is a computing environment that combines multiple public cloud and private cloud services and their infrastructures to create a single, effective and resilient framework. Which enables the organization to fulfil its workload and its business objectives with less cost and more effective job. Amazon, Google, Cisco, Microsoft etc. are hybrid cloud providers.

\section{Virtualization}

With the help of virtualization technology [7], [8] we can connect multiple client machines simultaneously and at the same time. Virtualization is a technology that has transformed the fundamental way of cloud computing from a revolutionary thud. It basically creates virtual images of servers, operating systems, storage, networks, and application resources, etc. thereby reducing the cost and energy on machine hardware. The virtualization concept is not new, it has existed since the 1960s, when IBM first created virtual machines to access replicated interfaces in mainframe computers [9] The real objective of virtualization is to change the way of traditional computing to make it more scalable, efficient, and economical. There is still a lot of work going on in this area so that our computing workload can be reduced and managed easily. Virtualization works on a software layer called a hypervisor, in which the virtual machine acts as a complete computer 
hardware with virtualized CPUs, RAM and I/O devices.

Hypervisor: Virtualization has an important role in cloud computing and with the help of this virtualization technology creates virtual machines. Now software is required to create and run this virtual machine on a computer, this software is known as hypervisor. It can also be in the form of hardware or firmware that works on the software layer of the operating system of the host machine. The computer on which more than one virtual machine is run with the help of a hypervisor is called the host machine and the virtual machine is the guest machine. The hypervisor is used in two ways: first is Type-1 hypervisor, also known as native hypervisor. This hypervisor can run directly a virtualized machine on a host machine. Type-2 hypervisor or hosted hypervisor is run on the software layer of the operating system of the host machine, just like other computer programs.

The Type-1 or native hypervisor can directly access the hardware resources of the host machine, so Type1 or native hypervisor provides greater efficiency and scalability than Type-2 hypervisor.

\section{Type of Virtualization}

Type of virtualization over cloud computing [10] are following-

1) Storage virtualization: Cloud storage itself is called storage virtualization, in which physical storage is arranged virtually in the cloud, and from there, multiple devices provide storage as per the requirement. Storage virtualization technology [11] has changed the way we store our data, earlier we had to carry a limited storage size hard disk which was less scalable and had some security issues. Today, the cloud has multiple users' data stores and users can access their data at anytime from anywhere with the help of the internet. Use of personal smart phones at work in organizations made Virtual Mobile Infrastructure [12], [13] a reality and nowadays increasing their popularity.
2) Software virtualization: Software virtualization in cloud computing greatly benefits the IT environment. It allows to create multiple virtual environments on a single computer system. In this virtual environment, with the help of some software such as VMware, Virtual Box etc. one or more operating systems can be installed and run on the same computer system and it gives to users the impression of a single and complete computer. Software virtualization has made the operating system and application testing process easier. This allows users to test other operating systems and applications on the same computer system as on the virtual operating system. Software virtualization provides flexibility to users as well as security such as firewall protection so that the user can safely make changes in other operating systems or applications according to some modification or requirement, on a single system.

3) Network virtualization: In network virtualization, multiple virtual sub-networks can be created on the same physical network, which can control this entire virtual network from a single software-based network administrator's console. The main objective of designing network virtualization in the IT environment is to speed up application delivery, increase security, boost IT productivity, optimize data transfer rates, and increase network flexibility. Virtual LAN (VLAN) can be a great example of network virtualization, which is the only part of the LAN that has been created with the help of software. Virtual LAN connects all network devices together into a group to improve the performance and speed of the network.

There are currently two types of network virtualization:

External Network Virtualization: In external network virtualization, we include several external local networks, in which to improve network efficiency and speed, all of these local networks combine to form a single virtual network. 
Internal Network Virtualization: This network virtualization is entirely based on software, in which with the help of software to create virtual networks in the existing network and connect multiple devices. The biggest advantage of this type of software-based network virtualization is that it does not depend on the hardware and also provides features like virtual networking, virtual switching and virtual firewalls.

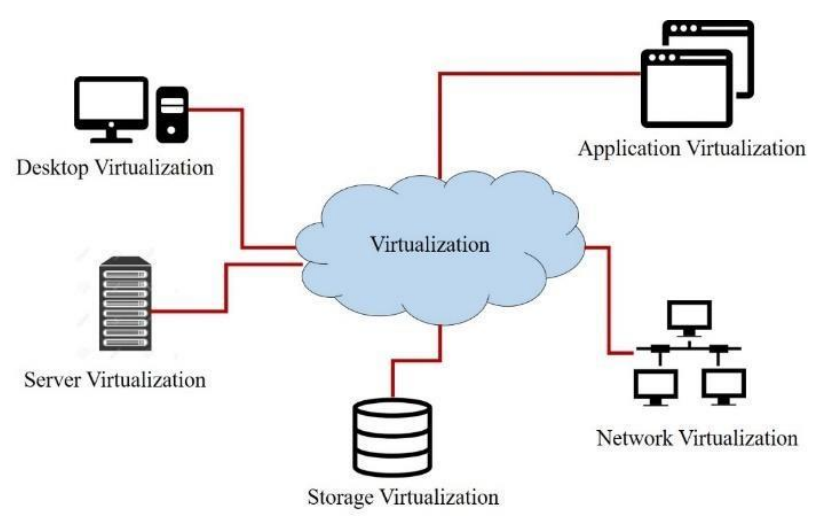

Figure 1. Type of Virtualization

4) Server Virtualization: Server virtualization [14] is leveraging the IT sector organization in many ways, with the first and important advantage being integrating their servers. The server virtualization supports multiple virtual machines. This means that multiple organizations can share their data from a single physical centralized server, which takes into account costs such as labour costs, power facility costs, floor space and cooling on a single physical server.

Why virtualization? Virtualization benefits the IT sector and organization in many ways and helps in the utilization and expansion of available resources. Virtualization is useful for several reasons, which are describe in detail:

Separation between users: The user is afraid of his data or information being stolen or used by another user, so it will be necessary to avoid the fear that all users should be separated from each other which is possible with the help of virtualization.
Resource fragmentation: In order to expand an available and large resource, it would be necessary to divide the large resource into smaller virtual resources with the help of virtualization techniques so that more and more users can share and take advantage of the resource.

Resource reallocation: It is a challenge to reallocate large resources such as storage, computation etc., so such a large resource can be easily reallocated by partitioning it into a small virtual resource with the help of virtualization.

Resource aggregation: To make the resources accessible to all users, it is necessary to expand that resource widely so that the user can use these resources from anywhere to reduce their workload and work time. With the help of virtualization, it can be expanded by splitting the resources into smaller virtual resources.

\section{5) Desktop Virtualization: Desktop virtualization}

[15] is a software-based technology that allows the user to connect remote devices without any specific hardware and also allows them to access their data and applications. The main aim of desktop virtualization technology is to reduce the user's cost of frequent upgradation of their computer system [16] In desktop virtualization technology [17], the user's computer system can virtually connect to a huge centralized server through a network. And it allows one to access virtual CPUs or RAMs to convert his/her old PCs into new updated PCs with new experience. To achieve desktop virtualization usually depends on two things: The operating system is running locally or remotely, which we call as Local Desktop Virtualization and Remote Desktop Virtualization respectively. In Local Desktop Virtualization, the operating system runs on local devices with the help of hardware virtualization and all operations are performed locally on the client 
device. In Remote Desktop Virtualization [18], the client and server, also called data centres, are interconnected with the help of the internet and the client's thin devices such as laptops and smartphones run the operating system and applications inside the data centre.

\section{E. Benefits of Desktop Virtualization}

Desktop virtualization has proved to be an important and valuable technology for large and small organization in IT environment that solves many of their problems and also benefits their organization's systems in many ways, out of which four major benefits categories are mentioned below-

1) Cost Savings: Desktop virtualization reduces the cost of frequent upgradation of the organization's computer systems, as well as its maintenance, management and support cost. According to experts, the maintenance and management cost of the organization's old computer's hardware and software is 50 to 60 percent of the total cost of ownership of the computer, which is a costly deal for the organization. That's why many companies and organizations have started giving importance to virtual desktop more than computer renewal.

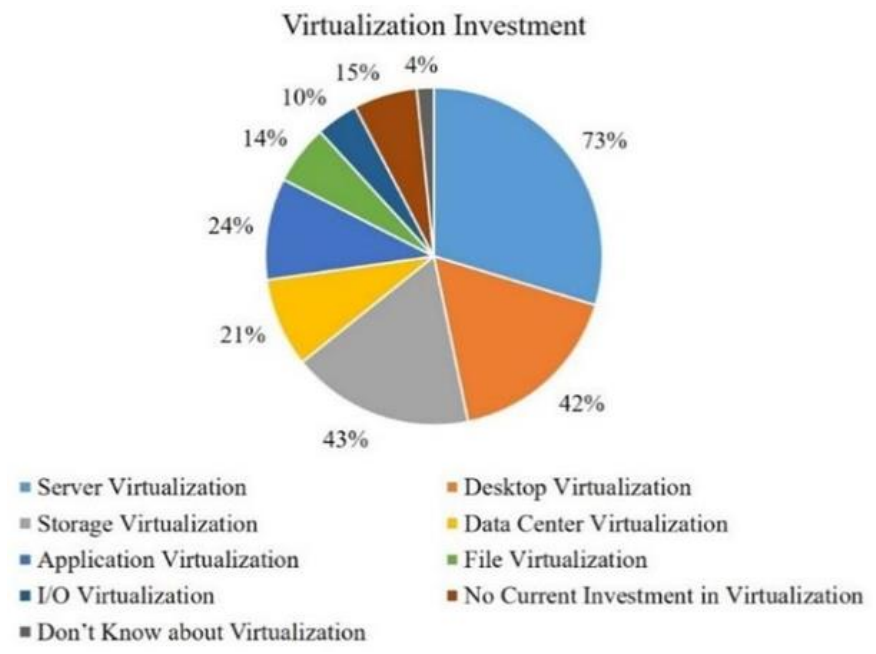

Figure 2. Virtualization Investment
2) Simplified Management: The data of the virtual desktop is not stored on the local machine, rather all the data is stored on a large centralized data centre. Since everything such as the operating system, application and data of the virtual desktop is centralized, it becomes easier for the client to maintain and manage their machine. Being centrally managed reduces client machine requirements such as frequently updating software, backing up files and scanning viruses, and more. Virtual desktop helps the client to use the old machine and extend its life to a great extent.

3) Enhanced Security: Many organizations and companies in the IT environment use desktop virtualization technology to avoid problems such as theft, tampering and loss of their confidential information and data. Because users do not keep confidential data on their personal device, all these data are managed in a data centre which is organized by the same company or third-party companies. And if the user loses or breaks his personal device, then that data can be backed up again from data centre.

4) Increased Productivity: Desktop virtualizations provide users freedom from working in one place and on the same machine. Because with the help of desktop virtualization technology, users are allowed to access applications and data from any other machine. If a user's machine breaks down, then he can log in to another machine with the help of his user id and password and continue his work without stopping. It can use any machine such as computers, tablets, and smartphones etc., because all the data is not stored on the local machine, rather stored in a data centre which is managed centrally. Having all the data centrally managed to allows the users to work from any corner of the globe which saves the down time and workload. 


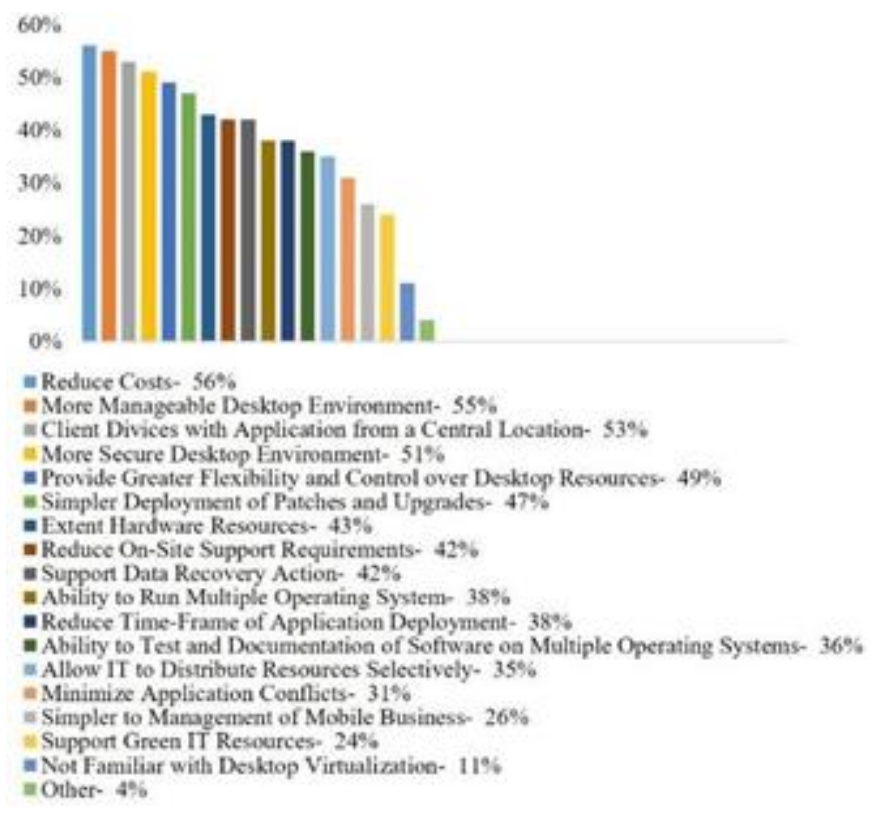

Figure 3. Benefits of Desktop Virtualization

\section{F. Challenges in Desktop Virtualization}

1) Network strength for good graphics: In desktop virtualization all virtual desktops are connected with cloud data centres over the network i.e. internet even distance between data centre and its users are very far. Therefore, it is important to have good network strength as well as a good algorithm to access rich graphics data from a long distance over the internet.

2) Network stability: Network stability plays an important role in desktop virtualization. Because the network must be stable when accessing data. If the network is not stable, then our data may be lost forever.

3) Network speed: In desktop virtualization, all virtual desktops are connected to the network, so it is necessary to have network speed to access data fast. Otherwise, we will not get rich graphics of data, network's slow speed will increase the chances of loss of data to many extents. If the network speed is slow, then related work will also be slow, which will be time consuming challenges.

4) Uncertainty of ROI: In desktop virtualization, users have to face the problem of uncertainty of ROI. Cost of virtualized desktop and its server maintenance, network upgradation, data storage, new thin-client devices affect the ROI. Therefore, it is important to improve the management to never affect the user's ROI factor like

- Centralized Management.

- Improve desktop security.

- Low power consumption with thin- client.

- Fast service for desktop deployment.

- Improve Network Strength.

- Upgrade OS and application with less downtime.

5) Require significant bandwidth: Users receive their data through the network from the data center. Therefore, it is necessary that the bandwidth of the network is low. So that, the user does not have the problem of data- traffic in accessing the data and at the same time is able to access his data and information in a short time. It is a big and expensive challenge for desktop virtualization, reducing their network bandwidth. Therefore, it is important to have an algorithm design for better performance of desktop virtualization that supports low and single bandwidth.

6) Performance: In desktop virtualization thin-client devices are connected with data centres over the network. Therefore, network strength and network speed affect overall performance of desktop virtualization systems. In which the main performance issue has been lag time to access the data, so performance is a big challenge to the famous desktop virtualization system in the IT environment. Therefore, it is necessary to make an algorithm to reduce network congestion when multiple users access the same data centre.

7) Security: Security is the biggest challenge of desktop virtualization systems. Every user has sensitive data and never likes to compromise with his data security. Therefore, it is necessary to prioritize the security of the user's data in the desktop virtualization system which is present on the centralized server. The main issue of security is lost or steals the data from the server during server maintenance or failure of the data server. So, it is 
important to back up the user's data at least one or more locations. So that, users can access the data during server maintenance or failure of server from backup location of his data.

\section{RELATED WORK}

In the work of [16] Dr. Jim Metzler presents benefits, challenges and its solution of the three forms of virtualization:

\section{- Server virtualization.}

- Desktop virtualization.

- Virtualized Appliances.

We consider desktop virtualization in details of benefits, challenges and its solutions. According to (Metzler, 2011), desktop virtualization is a technology that allows IT organizations and companies to transform their computer desktops into multiple virtual desktops and interconnect them and perform centrally single maintenance. Desktop virtualization can be obtained from many approaches, in which two main approaches are:

Server-Based Computing (SBC): Many organizations and companies used the SBC approach for a long time to achieve desktop virtualization which is similar to terminal based and time-shared multi-user computer systems.

Virtual Desktop Infrastructure (VDI): This is a new way of obtaining server-side virtualization which requires a $\mathrm{VM}$ on a central server to host a single virtual desktop.

Dr. Jim Metzler analysed the data from the Interope Survey to explain the adoption of desktop virtualization and stated that there are several factors to adopt desktop virtualization, the main factors being:
- Reduction in Overall cost.

- Reduction in OPEX cost for maintenance and support.

- Upgradation in security rules and its technology.

- Improvement in security of data and application.

In the use of desktop virtualization, if you talk about the challenges, the first challenge will be its performance and user experience for communication or connection of client to server over a wide range network like WAN.

The work of [16] has been introduced about applications have used ICA and RDP protocol to support compression techniques like bitmap compression, screen refresh compression, and general data compression to allow to easily communicate with remote client and server over WAN but challenges is, this type of application achieve better performance with only traditional data application but they have limitations with some data like rich graphics application, 3D graphics, audio and video. To overcome this type of limitation, applications like XenDekstop 4 use High-Definition user eXperience (HDX) technology that provides rich graphics application, video, audio and 3D graphics to remote desktop over a wide range network.

The main objective of the paper [5] is to save power consumption in IT sectors as well as to reduce the overall energy cost. This paper [5] has Introduced Grinfy system, under which IT companies or organizations can save at least $30 \%$ of the energy used on their computer system without Virtual Desktop Infrastructure (VDI). This paper mentions the Citrix and MacAfee research survey, according to survey VDI stands many benefits to IT and business organization, like:

- VDI reduces the operational cost.

- Enhances the flexibility of desktop Application.

- Provides better performance. 
- Increase the user experience and give more satisfaction to users.

- Provides more security for the desktop data.

According to experimental data of Grinfy System, VDI stands important role in users' desktop for save the power consumption. Experimental data of Grinfy System, presents the role of VDI in Grinfy System and gives benefit to IT sector and organization, if they use Grinfy without VDI then they can save at least 30\% of power consumption and save up to $90 \%$ with VDI.

This paper [19] has been presented about the BIM Cloud System Framework which is based on VDI technology which has proved very useful in construction planning. Many projects of BIM can be improved by taking advantage of Desktop Virtualization. However, some problems are encountered while starting BIM which slows down its expansion in the construction industry, problems are:

- Greater Infrastructure Development Cost.

- BIM software compatibility issues.

- Problems with determining the BIM process.

- Shortage of experts of BIM's tools.

- Lack of standards and guidelines of using BIM.

But [19] says that BIM Cloud Framework with VDI technology will prove to be very successful in construction projects over time and will also help in construction projects to reduce workload. Because, BIM has a lot of advantages which will make it important in construction industries as well as give a boost to its expansion:

- Reduced the work time of projects.

- Reutilization of extensive project data like design or map.

- Completion of work by skilled workforce.

- Increments in design quality.

- Reduced the error and changes associated with construction work.
According to [20], demand for desktop virtualization is becoming more and more in the IT sector due to data and desktop devices' proliferation. The main reason for the expansion of desktop virtualization may be to seek the help of remote IT workers.

The paper [21] is a detailed description of the desktop virtualization technique, which mainly reflects the implementation of VDI infrastructure, which allows the client's operating system to run on a centralized server-based virtual machine. Talking about implementation of VDI, static and dynamic are its two core architectures. In a static architecture, a single system has a unique virtual machine, that is, each user has to create and maintain a virtual machine which is good for security but it is a costal deal that affects the company's total cost expenditure. Whereas dynamic architecture supports server-based desktop virtualization in which multiple virtual machines run simultaneously on server. Users access virtual machines as per requirement with the help of hypervisors. According to this paper desktop virtualization provides benefits to users in many ways, such as reduced maintenance complexity and its overall cost, provide users flexibility, simplify administration, and more. Hence, before implementing this, the users should ensure which needs and benefits to be achieved. Therefore, according to this, its technology should be implemented.

Application streaming technology [22] has been used to achieve a viable cloud computing in which removing all barriers between clients and computing resources with the help of application streaming technology and to achieve a better physical resource management and performance. [22] proposes a lightweight virtualization system to achieve high performance of virtual systems, which has studied the main framework of the system model to achieve it. Application streaming, which is used to create images of application and host image on servers to achieve a 
virtualized desktop and make all images available on the network so that users can easily get the virtual desktop by accessing the image through the network. In the system model, Remote Order Transmission (ROT) protocol is designed to allow more interaction between the server and the client.

Converting physical servers into Virtual Server Infrastructure (VSI) and desktops into Virtual Desktop Infrastructure (VDI) has to face problems and impacts in areas such as server consolidation, performance, total cost of ownership (TCO), and return of investment (ROI). The purpose of the work in [23] is to clarify the costs and benefits of virtualization infrastructure. If we talk about the costs then mainly [23] has explored TCO and ROI, which is affected due to many reasons. The main reason is to transition from physical (server, storage, application, etc.) to virtual. Therefore, this paper has done an empirical analysis based on performance and highest $\backslash$ lowest TCO \& ROI by installing five different hypervisors such as VMware ESXi 5.0 (Redhat Linux), MS Hyper-V R2 (Windows Server 2008), Proxmox VE (Debian), KVM (Ubuntu Enterprise Server), and Xen (Cent OS) on different virtual servers in VSiC. As a result of empirical analysis Hyper-V performs well overall and ESX servers achieve highest ROI and lowest TCO in server virtualization but costs of these two are very high. Proxmox VE performs well with lowest c/p ratio and better ROI, which is a good choice for desktop and server virtualization.

According to [24], a traditional desktop environment is costly and managing its task like- Installation, changes of its configuration, requires time consuming procedures for security measures is very challenging. [24] focuses on methods and tools to transition legacy desktops into cloud desktops. In which two main tools are described, User Profiling (UPROF) and Desktop Benchmarking (DeskBench). UPROF is a user workload analysis tool that collects the fine details of the users' desktop and analyzes the processes of the system in detail. The feature of the UPROF tool is that it keeps collecting data even after it is disconnected from the network, it does not matter whether the users' desktop is connected to the network or not. UPROF collects all the data in a time interval of 10 seconds and then uploads the data on the collection server as soon as it is connected to the network so that the communication between the users' desktop and the server is continuous. The DeskBench tool records the actions of users' desktop and processes it in an orderly manner and then injects the events or actions of users' desktops to the server. The DeskBench tool collects and analyzes the users' desktop actions such as keyboard and mouse events, file locations, and more. The DeskBench tool works in two phases, the first being the Recording phase, in which events such as keyboard and mouse clicks are recorded and then saved in the artifact file. The second phase is the Replaying phase, in which it searches mouse and keyboard events saved in the artifact file, then injects the action sequentially to Windows Manager on the server.

\section{COMPARATIVE ANALYSIS OF APPROACHES}

In this section, the early work has been compared on the basis of five parameters- security, resource utilization, power consumption, execution cost, and allocation time as shown in table-1. Each parameter has got some scale value 1 to 10 according to his performance. Higher scale value of parameter indicates his better result. There are six columns in this table-1, the first column represents the author's approaches and the next five columns represent the parameters of the approaches.

Data of the given table-1 represent the magnitude of the parameter of approaches. Every approach performs better in Resource utilization, Execution cost, and Allocation time column, average performance in security and poor in power consumption. According to table data conclusion, 
power consumption is a more challenging parameter of desktop virtualization systems because it has no good algorithm in VDI technology to use it.

TABLE I. COMPARISON OF RELATED WORK WITH FIVES PARAMETERS

\begin{tabular}{|c|c|c|c|c|c|}
\hline Paper & $\begin{array}{l}\text { Sec } \\
\text { urit } \\
y\end{array}$ & $\begin{array}{l}\text { Resour } \\
\text { ce } \\
\text { Utilizat } \\
\text { ion }\end{array}$ & $\begin{array}{l}\text { Power } \\
\text { Consu } \\
\text { mption }\end{array}$ & $\begin{array}{l}\text { Execu } \\
\text { tion } \\
\text { Cost }\end{array}$ & $\begin{array}{l}\text { Alloc } \\
\text { ation } \\
\text { Time }\end{array}$ \\
\hline $\begin{array}{l}\text { Virtualization } \\
\text { Solution [16] }\end{array}$ & 7 & 7 & 1 & 6 & 4 \\
\hline $\begin{array}{l}\text { Grinfy with VDI } \\
\text { [5] }\end{array}$ & 5 & 5 & 9 & 7 & 3 \\
\hline $\begin{array}{l}\text { BIM with VDI } \\
{[19]}\end{array}$ & 4 & 6 & 1 & 7 & 5 \\
\hline IDG Survey [20] & 3 & 5 & 1 & 6 & 4 \\
\hline $\begin{array}{l}\text { Demystify of } \\
\text { VDI [21] }\end{array}$ & 3 & 5 & 1 & 4 & 5 \\
\hline $\begin{array}{l}\text { Application } \\
\text { Streaming [22] }\end{array}$ & 4 & 9 & 3 & 5 & 6 \\
\hline $\begin{array}{l}\text { VSiC } \\
\text { Architecture } \\
{[23]}\end{array}$ & 5 & 7 & 1 & 7 & 6 \\
\hline $\begin{array}{l}\text { UPROF \& } \\
\text { DeskBench [24] }\end{array}$ & 4 & 6 & 1 & 5 & 5 \\
\hline
\end{tabular}

Virtualization solutions [16] explore challenges and their solutions. [16] has to improve two fundamental forms of desktop virtualization. First is server-side desktop virtualization and second is client-side desktop virtualization security in the field of security and also utilized its resources well. Talking about power consumption, this paper has not given any solution for power consumption.

Power consumption can be saved by using Grinfy system [5] in VDI technology. Other parameters such as security, resource utilization, and execution cost and allocation time have also benefited from its use. Grinfy systems can save power consumption in VDI technology from $30 \%$ to $40 \%$.
BIM technology [19] has proved very useful for construction projects. BIM technology has greatly reduced execution cost and allocation time and has

helped in routing and improving the quality of the extensible design data.

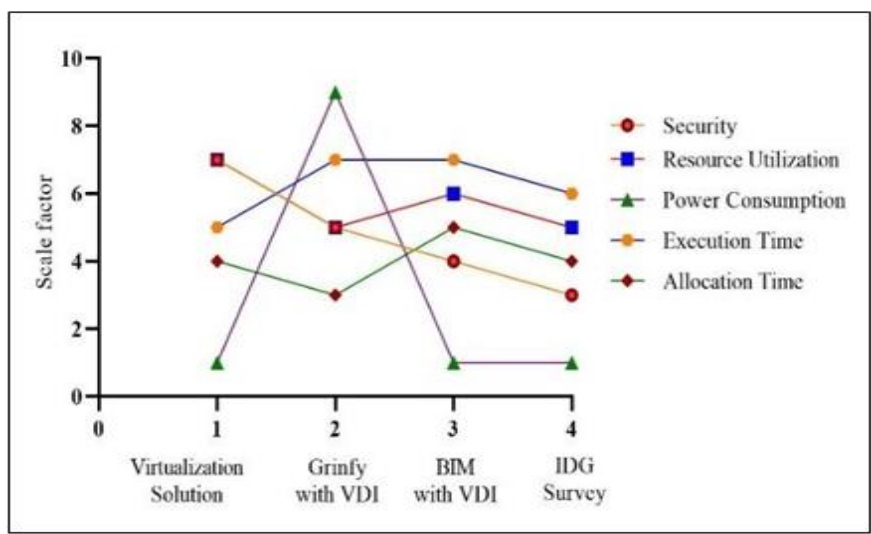

Figure 4. Comparative analysis of approaches: 1-4

International Data Group (IDG) survey [20] introduced us to the client side and server-side benefits and challenges of desktop virtualization, and accordingly installed hypervisor applications on client desktops to access multiple operating systems.

Which affects execution cost, security resource utilization and allocation time in a good direction.

Demystify of VDI [21] introduced an understanding concept of virtualization techniques and new architecture model of desktop virtualization like static and dynamic two core architecture of VDI. It helps to improve security as well as execution cost and allocation time. This architecture model doesn't save the power consumption of desktop virtualization systems.

Make desktop virtualization systems lightweight using Application Streaming technology [22]. This paradigm improves data security, execution cost, as well as flexibility of desktop virtualization. 
Application streaming technology is used to get lightweight virtualization systems and to achieve high performance and better resource management.

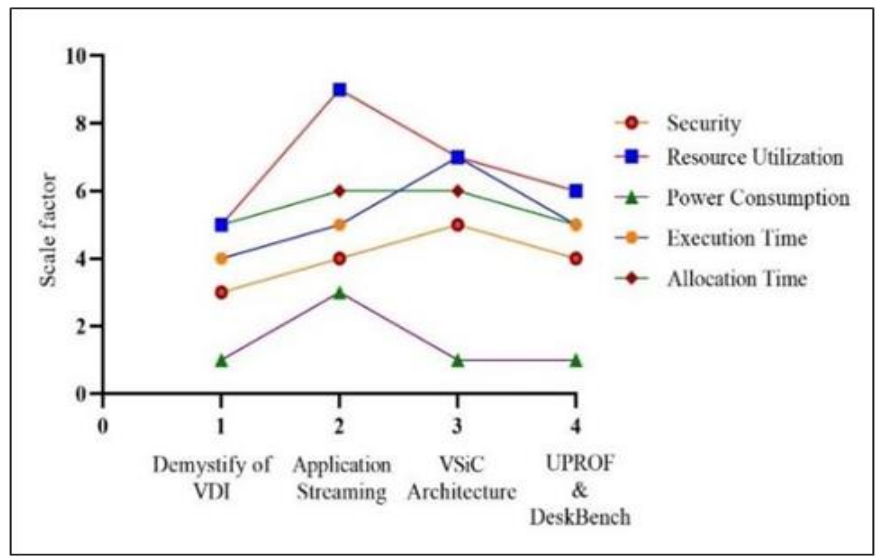

Figure 5. Comparative analysis of approaches: 5-8

VSiC architecture [23] has been very keen on improving virtual servers based on parameters such as security, execution cost and performance. VSiC architecture has strengthened the ratio of Total Cost of Owner (TCO) and Return of Investment (ROI) and performed well in execution cost and allocation time by utilizing resources. And in contrast, performance in the field of power consumption was poor.

With the help of UPROF and DeskBench [24], and VDI [25] legacy desktops are transformed efficiently and accurately into the desktop cloud. This makes users to make the device lighter and data security a step further. UPROF and DeskBench improves the resource utilization of the desktop users by obtaining the fine grain details of the system processes and application processes. Furthermore, there is no loss in over-all performance of the system.

\section{CONCLUSION}

Desktop virtualization is expanding rapidly in the IT sector. This is very useful for IT organization. As discussed in preceding sections, desktop virtualization is very useful for the users and for the business growth due to its economic advantages. Apart from the number of advantages, desktop virtualization also limits with various challenges discussed in paper. The paper aims to present the comparative analysis of various approaches for desktop virtualization to offer the best approach to the users on desktop virtualization. Comparative analysis section stating that Virtualization Solution approach better perform in security parameter and this approach promise to give more security during virtualization. Second approach Grinfy with VDI is well perform in power consumption parameter of VDI which offer save power up to 30 to 40 percent. Resource utilization parameter better perform in Application Streaming technology which maximize the utilization of physical resources. Performance of execution cost is good in three approaches Grinfy with VDI, BIM with VDI, and VSiC architecture. These three approaches promise to reduce the execution cost of desktop virtualization. Application Streaming and VSiC architecture are similar perform in allocation time parameter, these approaches reduced the allocation time and make the system flexible and scalable. But no-one approach is performing on all the parameters. So, in our future work, we will try to present an approach which will perform well on security, resource utilization and power consumption and execution cost and allocation time. Successful solution on desktop virtualization will offer the economic benefits to users and business persons with high end performance.

\section{REFERENCES}

[1]. Ibrahim, Abdallah Ali ZA, Dzmitry Kliazovich, Pascal Bouvry, and Ariel Oleksiak. "Virtual Desktop Infrastructures: architecture, survey and green aspects proof of concept" Seventh IEEE International Green and Sustainable Computing Conference (IGSC), pp. 1-8, 2016.

[2]. Swathi, T., K. Srikanth, and S. Raghunath Reddy. "Virtualization in cloud computing" International Journal of Computer Science and 
Mobile Computing, vol 3, No. 5, pp. 540-546, 2014.

[3]. Thakral, Deepanshu, and Mahesh Singh. "Virtualization in cloud computing" International Journal of Computer Science and Mobile Computing, Vol. 3, pp. 1262-1273, 2014.

[4]. Mahbub-E-Noor, Md. "Virtualization and Cloud Computing", pp. 1-34, 2014.

[5]. Ibrahim, Abdallah Ali Zainelabden Abdallah, Dzmitry Kliazovich, Pascal Bouvry, and Ariel Oleksiak. "Using virtual desktop infrastructure to improve power efficiency in grinfy system", IEEE International Conference on Cloud Computing Technology and Science (CloudCom), pp. 85-89, 2016.

[6]. Yao, Qiufeng, Yanbo $\mathrm{Wu}$, and Jiangming Gao. "Research on application of cloud desktop virtualization for computer laboratories in universities" In IOP Conference Series: Materials Science and Engineering, vol. 563, no. 5, p. 052028. IOP Publishing, 2019.

[7]. Sharma Shruti, Sharanjit Singh, Amardeep Singh, and Ramanpreet Kaur, "Virtualization in Cloud Computing" International Journal of Scientific Research in Science, Engineering and Technology, vol. 2(4): pp. 181-186, 2016.

[8]. Susanta, Nanda, and Chiueh Tzi-Cker. "A survey on virtualization technologies", Experimental Computer Systems Lab 2005.

[9]. Obasuyi, Gabriel Cephas, and Arif Sari. "Security challenges of virtualization hypervisors in virtualized hardware environment" International Journal of Communications, Network and System Sciences, vol. 8, no. 07, pp. 260-273, 2015.

[10]. Wilde, Norman, and Thomas Huber. "Virtualization and Cloud Computing" University Of West Florida, 2009.

[11]. Rosenblum, Mendel, and Carl Waldspurger. "I/O Virtualization: Decoupling a logical device from its physical implementation offers many compelling advantages" Queue 9, no. 11, pp. 3039, 2011.

[12]. Su, K., Liu, P., Gu, L., Chen, W., Hwang, K. and Yu, Z., "vMobiDesk: Desktop Virtualization for Mobile Operating Systems". IEEE Access, Vol. 8, pp. 213541-213553, 2020.

[13]. Adeliyi, Timothy T., and Oludayo O. Olugbara. "Optimizing Remote Access Using Mobile Cloud Virtual Desktop Infrastructure." IEEE Conference on Information Communications Technology and Society, pp. 1-4, 2021.

[14]. Jang, Su Min, Won Hyuk Choi, and Won Young Kim. "Client rendering method for desktop virtualization services" Etri Journal 35, no. 2, pp. 348-351, 2013.

[15]. Ameen Radhwan Y, and Hamo Asmaa Y, "Survey of Server Virtualization", International Journal of Computer Science and Information Security, Vol. 11, No. 3, pp. 1-10, 2013.

[16]. Metzler, Jim. "Virtualization: benefits, challenges, and solutions" Riverbed Technology, San Francisco, pp. 1-24, 2011.

[17]. Hodgman, Matthew R. "Desktop virtualization: Applications and considerations" Contemporary Issues in Education Research (CIER) vol. 6, No. 1, pp. 123-132, 2013.

[18]. Xiong, Nian, Shan Zhou, Zujian Wu, and Zhen Zhang. "Design and research of hybrid cloud desktop scheme in colleges and universities." In MATEC Web of Conferences, vol. 336, p. 05004. EDP Sciences, 2021.

[19]. Lee, K. H., S. W. Kwon, J. H. Shin, and G. S. Choi. "A Study on Design of Virtual Desktop Infrastructure (VDI) System Model for Cloud Computing BIM Service" In ISARC. Proceedings of the International Symposium on Automation and Robotics in Construction, IAARC Publications, vol. 32, p. 1-6, 2015.

[20]. Santosus, Megan. "The Promise of Desktop Virtualization" IDG White Paper, http://sites. amd. 
com/us/Documents/promiseofdesktopvirtualizat ion.p df, pp. 1-6, 2008.

[21]. Petrović, Tomislav, and Krešimir Fertalj. "Demystifying desktop virtualization" In Proceedings of the 9th WSEAS international conference on Applied computer science, pp. 241-246. 2009.

[22]. Lai, Guangda, Hua Song, and Xiaola Lin. "A service based lightweight desktop virtualization system" IEEE International Conference on Service Sciences, pp. 277-282, 2010.

[23]. Chang, Bao Rong, Hsiu-Fen Tsai, and Chi-Ming Chen. "Empirical analysis of server consolidation and desktop virtualization in cloud computing" Mathematical Problems in Engineering, 2013.

[24]. Beaty, Kirk, Andrzej Kochut, and Hidayatullah Shaikh. "Desktop to cloud transformation planning" IEEE International Symposium on Parallel \& Distributed Processing, pp. 1-8, 2009.

[25]. Zhang, Kai. "Application of Desktop Computing Technology Based on Cloud Computing." International Journal of Information Technologies and Systems Approach" Vol. 14, No. 2, pp. 1-19, 2021.

\section{Cite this article as :}

Rupesh Kumar, Arun Kumar Yadav, H N Verma, "An analysis of Approaches for Desktop Virtualization and Challenges", International Journal of Scientific Research in Computer Science, Engineering and Information Technology (IJSRCSEIT), ISSN : 24563307, Volume 7 Issue 4, pp. 600-612, July-August 2021. Available at doi : https://doi.org/10.32628/CSEIT2174133 Journal URL : https://ijsrcseit.com/CSEIT2174133 